\title{
Generation of Random Variates using Asymptotic Expansions
}

\author{
Jens Struckmeier \\ Department of Mathematics \\ University of Kaiserslautern \\ P.O Box 3049 \\ 67653 Kaiserslautern, Germany
}

AMS Subject Classfication: 65C10; 41A60.

Keywords: Random Number Generation; Aymptotic Expansions.

\begin{abstract}
Monte-Carlo methods are widely used numerical tools in various fields of application, like rarefied gas dynamics, vacuum technology, stellar dynamics or nuclear physics. A central part is the generation of random variates according to a given probability law. Fundamental techniques are the inversion principle or the acceptance-rejection method - both may be quite time-consuming if the given probability law has a complicated structure.

In this paper probability laws depending on a small parameter are considered and the use of asymptotic expansions to generate random variates is investigated.

The results given in the paper are restricted to first order expansions. Error estimates for the discrepancy as well as for the bounded Lipschitz distance of the asymptotic expansion are derived. Furthermore the integration error for some special classes of functions is given. The efficiency of the method is proofed by a numerical example from rarefied gas flows.
\end{abstract}

\section{Zusammenfassung}

Monte-Carlo Methoden sind weitverbreitete numerische Werkzeuge in verschiedenen Anwendungsbereichen, wie etwa der Theorie verdünnter Gase, Vakuumtechnologie, Stellardynamik oder Nuklearphysik. Ein zentraler Teil ist die Generierung von Zufallsvariablen nach einem vorgegebenen Wahrscheinlichkeitsgesetz. Grundlegende Methoden sind etwa die Umkehrmethode oder die Verwerfungsmethode, die beide sehr zeitaufwendig werden können, sofern das vorgebene Wahrscheinlichkeitsgesetz eine komplizierte Struktur besitzt.

In der vorliegenden Arbeit werden Wahrscheinlichkeitsgesetze studiert, die von einem kleinen Parameter abhängig sind, und es wird untersucht wie mit Hilfe asymptotischer Entwicklungen Zufallsvariablen erzeugt werden können.

Die Ergebnisse beschränken sich aus asymptotische Entwicklungen erster Ordnung. Es werden Fehlerabschätzungen sowohl für die Diskrepanz als auch den beschränkten Lipschitz Abstand der asymptotischen Entwicklungen gezeigt. Zusätzlich werden die Integrationsfehler für eine spezielle Klasse von Funktionen angegeben. Die Effizienz der Methode wird anhand eines numerischen Beispiels aus der Theorie verdünnter Gase bestätigt. 


\section{Introduction}

The generation of random variates according to a given density function $f$, the so-called $f$ distributed pointsets, is a crucial part in Monte-Carlo methods. General techniques - like the inversion principle or the rejection method - are given in the book of Devroye [2] together with a lot of concrete algorithms for special density functions.

In this paper we consider a density $f(x ; \varepsilon)$ which depends on a small parameter $\varepsilon>0$ and investigate the use of asymptotic expansions to generate $f$-distributed pointsets. We start our investigation with the following simple example:

Let

$$
f(x ; \varepsilon)=\frac{3}{3+\varepsilon}\left(1+\varepsilon x^{2}\right)
$$

be the density of a probability law on $[0,1]$ and $\varepsilon>0$ a small parameter.

We try to generate a sequence of random variables $\left\{x_{N}^{\varepsilon}\right\}_{N \in N}$ which are $f$-distributed using the inversion principle.

Denote by $F$ the distribution function of $f$, i.e.

$$
F(x ; \varepsilon)=\int_{0}^{x} f(t ; \varepsilon) d t
$$

Because $f(x ; \varepsilon)>0$ for all $x \in[0,1], F$ is strictly increasing and we are able to transform a uniformly distributed sequence $\left\{t_{i}^{N}\right\}_{N \in N} \subset[0,1]$ in a $f$-distributed sequence via the equation

$$
x^{\varepsilon}=F^{-1}(t ; \varepsilon)
$$

We compute

$$
F(x ; \varepsilon)=\frac{1}{3+\varepsilon} x\left(3+\varepsilon x^{2}\right)
$$

hence one has to compute the roots of a cubic equation to solve equation (3).

If $\varepsilon \ll 1$, one may consider $F(x ; \varepsilon)$ as a small perturbation of $F(x ; 0)$, with

$$
F(x ; 0)=x
$$

If we take the asymptotic expansion

$$
F(x ; \varepsilon) \sim x+\frac{\varepsilon}{3} x\left(x^{2}-1\right)
$$

and assume

$$
x^{\varepsilon} \sim x^{(0)}+\varepsilon x^{(1)}
$$

we get

$$
\begin{aligned}
& x^{(0)}=t \\
& x^{(1)}=\frac{1}{3} t\left(1-t^{2}\right)
\end{aligned}
$$

as second order approximation of the solution $x^{\varepsilon}$ of $F\left(x^{\varepsilon} ; \varepsilon\right)=t$.

Hence, instead of computing the roots of the cubic equation, we can take

$$
\bar{x}^{\varepsilon}=t+\frac{1}{3} \varepsilon t\left(1-t^{2}\right)
$$


and may expect that

$$
\left|x^{\varepsilon}-\bar{x}^{\varepsilon}\right|=o(\varepsilon)
$$

The expectation value of $x^{\varepsilon}$ is

$$
\begin{aligned}
\mathbb{E}\left(x^{\varepsilon}\right) & =\frac{3}{3+\varepsilon} \int_{0}^{1} x\left(1+\varepsilon x^{2}\right) d x \\
& =\frac{3(2+\varepsilon)}{4(3+\varepsilon)}
\end{aligned}
$$

and, if $\varepsilon \longrightarrow 0$

$$
\mathbb{E}\left(x^{\varepsilon}\right) \sim \frac{1}{2}+\frac{1}{12} \varepsilon+O\left(\varepsilon^{2}\right)
$$

On the other hand we have

$$
\begin{aligned}
\mathbb{E}\left(\hat{x}^{\varepsilon}\right) & =\mathbb{E}\left(x^{(0)}\right)+\varepsilon \mathbb{E}\left(x^{(1)}\right) \\
& =\frac{1}{2}+\frac{1}{12} \varepsilon
\end{aligned}
$$

\section{Remark 1}

Throughout the paper we will work with the measure theoretical aspect of $f$-distributed sequences on $\mathbb{R}$ :

We interpret the density $f$ of a probability law on $\mathbb{R}$ as the density of an absolutely continuous measure $\mu$ on $\mathbb{R}$ and consider a sequence of $f$-distributed pointsets $\left\{x_{1}^{N}, \ldots, x_{N}^{N}\right\}_{N \in N}$ as a sequence of discrete measures $\mu_{N}$ with

$$
\mu_{N}=\frac{1}{N} \sum_{i=1}^{N} \delta_{x_{i}^{N}}
$$

Using this notation we study the weak* convergence of $\mu_{N}$ to $\mu$. Furthermore we introduce distances between measures based on the fact that measures spaces are equiped with a metric structure.

In the next section we give some basic notations concerning asymptotic expansions and measure theory. In section 3 we will investigate first order expansions to generate $f$-distributed pointsets. Finally we present a numerical example.

\section{Basic Notations}

\section{Order Relations}

Definition 1 Suppose that $f(x)$ and $g(x)$ are two continuous functions on $\mathbb{R}$.

1) If there exists a constant $M$ and a neighborhood $N_{0}$ of $x=0$ such that

$$
f(x) \leq M \cdot g(x) \quad \forall x \in N_{0} \cap \mathbb{R}
$$

we say that, as $x \rightarrow 0$,

$$
f(x)=O(g(x))
$$


2) Suppose that for any $\varepsilon>0$ there exists a neighborhood $N_{\varepsilon}$ of $x=0$ such that

$$
f(x) \leq \varepsilon \cdot g(x) \quad \forall x \in N_{\varepsilon} \cap \mathbb{R}
$$

we say that, as $x \rightarrow 0$

$$
f(x)=o(g(x))
$$

\section{Asymptotic expansion of $f(x ; \varepsilon)$}

Definition 2 Let $f(x ; \varepsilon): \mathbb{R} \longrightarrow \mathbb{R}$ be a continuous function. The formal series

$$
\sum_{n=0}^{N-1} a_{n}(x) \varepsilon^{n}
$$

is called asymptotic sequence of $f$ with $N$ terms for $\varepsilon \rightarrow 0$, if

$$
\lim _{\varepsilon \rightarrow 0} \frac{f(x, \varepsilon)-\sum_{n=0}^{M} a_{n}(x) \varepsilon^{n}}{\varepsilon^{M}}=0
$$

holds for all $M<N$ and $x \in \mathbb{R}$.

\section{Remark 2}

1) Together with Definition 1 we have

$$
f(x ; \varepsilon)=\sum_{n=0}^{N-1} a_{n}(x) \varepsilon^{n}+o\left(\varepsilon^{N-1}\right)
$$

2) If the convergence in Definition 2 is uniformly with respect to $x$, we call the asymptotic expansion uniform.

3) If $f(\cdot ; \varepsilon)$ is differentiable up to order $N-1$ with respect to the parameter $\varepsilon$ at $\varepsilon=0$ then the asymptotic expansion (22) coincides with the Taylor expansion of order $N$-1, i.e.

$$
a_{n}(x)=f^{(n)}(x ; 0) \quad \forall n=0, \ldots, N-1
$$

In the following we consider probability measures $\mu$ on $(\mathbb{R}, \mathcal{B})$ where $\mathcal{B}$ denotes the $\sigma$-algebra of Borel sets on $\mathbb{R}$ and $\mu(\mathbb{R})=1$. We denote the space of such measures by $\mathcal{M}$.

Definition 3 A sequence $\left(\mu_{n}\right)_{n \in N} \subset \mathcal{M}$ is called weakly convergent to $\mu \in \mathcal{M}$, if

$$
\lim _{n \rightarrow \infty} \int \Phi(x) \mathrm{d} \mu_{n}=\int \Phi(x) \mathrm{d} \mu
$$

for all $\Phi \in \mathcal{C}_{b}(\mathbb{R})$.

Measure spaces are metric spaces hence one may introduce metrices on the space $\mathcal{M}$. We will use the following two metrices

1) the discrancy $D(\mu, \nu)$ and

2) the bounded Lipschitz distance $\rho(\mu, \nu)$. 


\section{Discrepancy}

Definition 4 Let $\mu, \nu \in \mathcal{M}$. Then the discrepancy $D(\mu, \nu)$ is given by

$$
D(\mu, \nu)=\sup _{x \in R}\left|\int_{-\infty}^{x} \mathrm{~d}(\mu-\nu)\right|
$$

Remark 3 The notion 'discrepancy' was introduced by H. Weyl in connection with the uniform distribution modulo 1 [5]. We denote the discrepancy of a uniformly distributed sequence $\omega_{N}=$ $\left\{t_{1}^{N}, \ldots, t_{N}^{N}\right\}$ on $[0,1]$ by $D\left(\omega_{N}\right)$.

The weak convergence of sequences is equivalent to the convergence of the discrepancy if the limit measure is absolutely continuous (a.c.).

Definition $5 \mu \in \mathcal{M}$ is called a.c., if their exists a non-negative function $f$ such that

$$
\int_{B} \mathrm{~d} \mu=\int_{B} f(x) \mathrm{d} x \quad \forall B \in \mathcal{B}
$$

We call the function $f$ a probability density on $\mathbb{R}$.

Remark 4 If the limit measure $\mu$ is a.c., we use the notation $D(f, \nu)$ instead of $D(\mu, \nu)$.

Theorem 1 [4]

Assume that $\mu \in \mathcal{M}$ is a.c., then the sequence $\left(\mu_{n}\right)_{n \in N} \subset \mathcal{M}$ converges weakly to $\mu \in \mathcal{M}$ iff

$$
\lim _{n \rightarrow \infty} D\left(\mu, \mu_{n}\right)=0
$$

The main advantage using the discrepancy is the Koksma inequality [5] which gives an error estimates for the integration of functions.

Theorem 2 Suppose $f$ is a probability density on $\mathbb{R}$ and $\left(\mu_{N}\right)_{N \in N} \subset \mathcal{M}$ a sequence of discrete measures. Then

$$
\left|\int_{R} \Phi(x) f(x) \mathrm{d} x-\frac{1}{N} \sum_{i=1}^{N} \Phi\left(x_{i}^{N}\right)\right| \leq V[\Phi] \cdot D\left(f, \mu_{N}\right)
$$

for all functions $\Phi: \mathbb{R} \longrightarrow \mathbb{R}$ which have a bounded variation $V[\Phi]$ on $\mathbb{R}$.

\section{Proof}

Using the proof of Niederreiter [5] for the unit intervall $[0,1]$ the generalization to measures on $\mathbb{R}$ is straightforward.

\section{Bounded Lipschitz distance}

Definition 6 Let $\mu, \nu \in \mathcal{M}$. Then the bounded Lipschitz distance $\rho(\mu, \nu)$ is defined as

$$
\rho(\mu, \nu)=\sup _{\Phi \in D}\left|\int_{R} \Phi \mathrm{d}(\mu-\nu)\right|
$$

where $D=\{\Phi: \mathbb{R} \longrightarrow \mathbb{R}, 0 \leq \Phi \leq 1,|\Phi(x)-\Phi(y)| \leq|x-y|\}$. 
Remark 5 If the limit measure $\mu$ is a.c., we use the notation $\rho(f, \nu)$ instead of $\rho(\mu, \nu)$.

Theorem 3 [3]

The sequence $\left(\mu_{n}\right)_{n \in N} \subset \mathcal{M}$ converges weakly to $\mu \in \mathcal{M}$ iff

$$
\lim _{n \rightarrow \infty} \rho\left(\mu, \mu_{n}\right)=0
$$

Concerning the integration error we have

Theorem 4 Let $\mu, \nu \in \mathcal{M}$. Then

$$
\left|\int_{R} \Phi(x) \mathrm{d}(\mu-\nu)\right| \leq 2 \max \{L, M\} \rho(\mu, \nu)
$$

for all functions $\Phi: \mathbb{R} \longrightarrow \mathbb{R}$ which are bounded by $M<\infty$ and Lipschitz-continuous with Lipschitz constant $L$.

\section{Proof}

Consider

$$
\left|\int_{\boldsymbol{R}} \Phi \mathrm{d}(\mu-\nu)\right| \leq 2\left|\int_{\boldsymbol{R}}\right| \Phi|\mathrm{d}(\mu-\nu)|
$$

and notice that $\bar{\Phi}=\frac{1}{\max \{L, M\}}|\Phi| \in D$. Hence

$$
\left|\int_{R} \bar{\Phi} \mathrm{d}(\mu-\nu)\right| \leq \rho(\mu, \nu)
$$

\section{First Order Expansions}

In this section we investigate asymptotic expansions of probability densities $f(x ; \varepsilon)$ with respect to the small parameter $\varepsilon>0$. We restrict ourselves to asymptotic expansions containing two terms, i.e.

$$
f(x ; \varepsilon) \sim a_{0}(x)+\varepsilon a_{1}(x) \quad \text { if } \varepsilon \rightarrow 0
$$

Furthermore we assume

$$
f(x ; \varepsilon)>0 \text { a.e. }
$$

The first problem is to derive an asymptotic expansion for the distribution function $F(x ; \varepsilon)$. If $f(x ; \varepsilon)$ can not be integrated explicitly we try to find the asymptotic expansion by integrating $a_{0}(x)$ and $a_{1}(x)$ :

\section{Theorem 5 Assume}

$$
f(x, \varepsilon) \sim a_{0}(x)+\varepsilon a_{1}(x)
$$

if $\varepsilon \rightarrow 0$, together with the properties

1) the functions $a_{0}$ and $a_{1}$ are a.e. continuous as well as $f(x ; \varepsilon)$ with respect to $x$ for all $\varepsilon>0$. 
2) there exists $F_{0} \in \mathcal{L}_{1}\left(\mathbb{R}, \mathbb{R}_{+}\right)$, such that

$$
f(x ; \varepsilon) \leq F_{0}(x) \quad \forall x \in \mathbb{R}, \varepsilon>0
$$

3) there exists $F_{1} \in \mathcal{L}_{1}\left(\mathbb{R}, \mathbb{R}_{+}\right)$, such that

$$
\left|\frac{f(x ; \varepsilon)-a_{0}(x)}{\varepsilon}\right| \leq F_{1}(x) \quad \forall x \in \mathbb{R}, \varepsilon>0
$$

Define the distribution function of $f(x ; \varepsilon)$ by

$$
F(x, \varepsilon)=\int_{-\infty}^{x} f(y, \varepsilon) \mathrm{d} y
$$

Then

$$
a_{0}, a_{1} \in \mathcal{L}_{1}(\mathbb{R})
$$

and

$$
F(x, \varepsilon) \sim A_{0}(x)+\varepsilon A_{1}(x)
$$

with

$$
A_{n}(x)=\int_{-\infty}^{x} a_{n}(y) \mathrm{d} y \quad n=0,1
$$

\section{Proof}

From Definition 2 we have

$$
\lim _{\varepsilon \rightarrow 0} f(x ; \varepsilon)=a_{0}(x) \quad \forall x \in \mathbb{R}
$$

and

$$
\lim _{\varepsilon \rightarrow 0} \frac{f(x ; \varepsilon)-a_{0}(x)}{\varepsilon}=a_{1}(x) \quad \forall x \in \mathbb{R}
$$

Using property 2) together with Lebesgue's Dominated Convergence Theorem yields

$$
a_{0}(x) \in \mathcal{L}_{1}\left(\mathbb{R}, \mathbb{R}_{+}\right)
$$

and

$$
\lim _{\varepsilon \rightarrow 0} \int_{-\infty}^{x} f(y ; \varepsilon) \mathrm{d} y=\int_{-\infty}^{x} a_{0}(y) \mathrm{d} y
$$

Hence we have

$$
F(x ; \varepsilon) \sim A_{0}(x) \quad \forall x \in \mathbb{R}
$$

Using property 3 ) we get with the same argument

$$
F(x ; \varepsilon) \sim A_{0}(x)+\varepsilon A_{1}(x)
$$

In order to explain how to use asymptotic expansions to generate $f$-distributed pointsets we consider again the inversion principle: 
If $\left\{t_{i}^{N}\right\}_{i=1, \ldots, N} \subset[0,1]$ is an uniformly distributed pointset one may determine a $f$-distributed pointset $\left\{x_{i}^{N}\right\}_{i=1, \ldots, N}$ via the relation

$$
F\left(x_{i}^{N} ; \varepsilon\right)=t_{i}^{N}
$$

Suppose $\varepsilon<\frac{1}{N}$ then we may consider the asymptotic expansion

$$
F(x ; \varepsilon) \sim A_{0}(x)+\varepsilon A_{1}(x)
$$

and try to construct a asymptotic expansion of the solution $x^{\varepsilon}$ of

$$
F(x ; \varepsilon)=t
$$

Taking the ansatz

$$
x^{\varepsilon} \approx x^{(0)}+\varepsilon x^{(1)}
$$

together with (52) yields

$$
A_{0}\left(x^{(0)}+\varepsilon x^{(1)}\right)+\varepsilon A_{1}\left(x^{(0)}+\varepsilon x^{(1)}\right)=t
$$

and

$$
A_{0}\left(x^{(0)}\right)+\varepsilon x^{(1)} a_{0}\left(x^{(0)}\right)+\varepsilon A_{1}\left(x^{(0)}\right)+O\left(\varepsilon^{2}\right)=t
$$

Comparing powers in $\varepsilon$ we get

$$
\begin{aligned}
A_{0}\left(x^{(0)}\right) & =t \\
x^{(1)} & =-\frac{A_{1}\left(x^{(0)}\right)}{a_{0}\left(x^{(0)}\right)} \quad \text { if } a_{0}\left(x^{(0)}\right) \neq 0
\end{aligned}
$$

Using this procedure we may expect that

$$
x^{\varepsilon} \sim x^{(0)}+\varepsilon x^{(1)}
$$

Theorem 6 Suppose

$$
F(x ; \varepsilon) \sim A_{0}(x)+\varepsilon A_{1}(x) \quad \text { if } \varepsilon \rightarrow 0
$$

uniformly with respect to $x$ and let $x^{\varepsilon}$ be the solution of

$$
F(x ; \varepsilon)=t \in[0,1]
$$

Define

$$
x^{(0)}=A_{0}^{-1}(t)
$$

Then

$$
x^{\varepsilon} \sim x^{(0)}-\varepsilon \frac{A_{1}\left(x^{(0)}\right)}{a_{0}\left(x^{(0)}\right)}
$$

\section{Proof}

First we have to show that $x^{\varepsilon} \sim x^{(0)}$ :

Because of $F(x ; \varepsilon) \stackrel{\varepsilon \rightarrow 0}{\longrightarrow} A_{0}(x)$ uniformly on $\mathbb{R}$ we have

$$
\left|F\left(x^{\varepsilon} ; \varepsilon\right)-A_{0}\left(x^{\varepsilon}\right)\right| \stackrel{\varepsilon \rightarrow 0}{\longrightarrow} 0
$$


Now

$$
\left|F\left(x^{\varepsilon} ; \varepsilon\right)-A_{0}\left(x^{\varepsilon}\right)\right|=\left|t-\left(A_{0} \circ F^{-1}\right)(t ; \varepsilon)\right|
$$

$A_{0}$ is continuous and strictly monotone hence $A_{0}^{-1}$ is a continuous function and

$$
\left|A_{0}^{-1}(t)-\left(A_{0}^{-1} \circ A_{0} \circ F^{-1}\right)(t ; \varepsilon)\right| \stackrel{\varepsilon \rightarrow 0}{\longrightarrow} 0
$$

respectively

$$
\lim _{\varepsilon \rightarrow 0}\left|x^{(0)}-x^{\varepsilon}\right|=0
$$

This completes the first part.

For the second part, i.e.

$$
\lim _{\varepsilon \rightarrow 0} \frac{x^{\varepsilon}-x^{(0)}}{\varepsilon}=x^{(1)}
$$

we use the following lemma.

Lemma 1 Let $\left(y_{\varepsilon}\right)$ be a sequence in $\mathbb{R}$ with $y_{\varepsilon} \stackrel{\varepsilon \rightarrow 0}{\longrightarrow} y$ and

$$
\frac{y-y_{\varepsilon}}{\varepsilon} \stackrel{\varepsilon \rightarrow 0}{\longrightarrow} c
$$

and $f \in \mathcal{C}^{1}(\mathbb{R})$. Then

$$
\frac{f(y)-f\left(y_{\varepsilon}\right)}{\varepsilon} \longrightarrow c f^{\prime}(y)
$$

if $\varepsilon \rightarrow 0$.

\section{Proof}

Because of (69) there exists a function $h(\varepsilon)$ such that

$$
y=y_{\varepsilon}+\varepsilon c+h(\varepsilon)
$$

with

$$
\frac{h(\varepsilon)}{\varepsilon} \longrightarrow 0 \quad \text { if } \varepsilon \rightarrow 0
$$

Consider the term

$$
\left|\frac{f(y)-f\left(y_{\varepsilon}\right)}{\varepsilon}-c f^{\prime}(y)\right|
$$

Using (71) yields

$$
\left|\frac{f(y)-f\left(y_{\varepsilon}\right)}{\varepsilon}-c f^{\prime}(y)\right|=\left|\frac{f(y)-f(y-\varepsilon c-h(\varepsilon))}{\varepsilon}-c f^{\prime}(y)\right|
$$

or

$$
\left|\frac{f(y)-f\left(y_{\varepsilon}\right)}{\varepsilon}-c f^{\prime}(y)\right|=\left|c\left(1+\frac{h(\varepsilon)}{\varepsilon}\right) \frac{f(y)-f(y-\varepsilon c-h(\varepsilon))}{\varepsilon c+h(\varepsilon)}-c f^{\prime}(y)\right|
$$

Because of $\varepsilon c+h(\varepsilon) \stackrel{\varepsilon \rightarrow 0}{\longrightarrow} 0$ and $(72)$

$$
\left|\frac{f(y)-f\left(y_{\varepsilon}\right)}{\varepsilon}-c f^{\prime}(y)\right| \stackrel{\varepsilon \rightarrow 0}{\longrightarrow} 0
$$


In order to apply Lemma 1 we first notice that

$$
\left|\frac{F\left(x^{\varepsilon} ; \varepsilon\right)-A_{0}\left(x^{\varepsilon}\right)}{\varepsilon}-A_{1}\left(x^{(0)}\right)\right| \stackrel{\varepsilon \rightarrow 0}{\longrightarrow} 0,
$$

(because

$$
\left|\frac{F\left(x^{\varepsilon} ; \varepsilon\right)-A_{0}(\varepsilon)}{\varepsilon}-A_{1}\left(x^{(0)}\right)\right| \leq\left|\frac{F\left(x^{\varepsilon} ; \varepsilon\right)-A_{0}(\varepsilon)}{\varepsilon}-A_{1}\left(x^{\varepsilon}\right)\right|+\left|A_{1}\left(x^{\varepsilon}\right)-A_{1}\left(x^{(0)}\right)\right|
$$

and both terms on the left side converge to 0 ).

Now introducing

$$
t^{\varepsilon}=A_{0}\left(x^{\varepsilon}\right)
$$

we may write equation (77) in the form

$$
\left|\frac{t-t^{\varepsilon}}{\varepsilon}-\left(A_{1} \circ A_{0}^{-1}\right)(t)\right| \stackrel{\varepsilon \rightarrow 0}{\longrightarrow} 0
$$

$A_{0}^{-1}$ is strictly increasing and differentiable hence using Lemma 1 we get

$$
\left|\frac{x^{\varepsilon}-x^{(0)}}{\varepsilon}+\frac{A_{1}\left(x^{(0)}\right)}{a_{0}\left(x^{(0)}\right)}\right| \stackrel{\varepsilon \rightarrow 0}{\longrightarrow} 0
$$

which completes the proof of the theorem.

Remark 6 Because $x^{\varepsilon} \sim x^{(0)}+\varepsilon x^{(1)}$ we have

$$
x^{\varepsilon}=x^{(0)}+\varepsilon x^{(1)}+o(\varepsilon)
$$

Example 1 Let us consider the simple example given in the introduction. The asymptotic expansion of the distribution function $F(x ; \varepsilon)$ with $x \in[0,1]$ is given by

$$
F(x ; \varepsilon) \sim x+\frac{\varepsilon}{3} x\left(x^{2}-1\right)
$$

Hence we directly get

$$
x^{\varepsilon} \sim t-\frac{\varepsilon}{3} t\left(t^{2}-1\right)
$$

The result of theorem 6 can be used to construct an 'asymptotic expansion' of an $f$-distributed pointset:

Suppose $\left\{t_{1}^{N}, \ldots, t_{N}^{N}\right\}$ is an uniformly distributed pointset on $[0,1]$, i.e.

$$
D\left(\omega_{N}\right) \longrightarrow 0 \quad \text { if } N \longrightarrow 0
$$

with

$$
\omega_{N}=\frac{1}{N} \sum_{i=1}^{N} \delta_{t_{i}^{N}}
$$

Then we may consider the discrete measure

$$
\mu_{N}=\frac{1}{N} \sum_{i=1}^{N} \delta_{x_{i}^{\varepsilon}}
$$


with

$$
F\left(x_{i}^{\varepsilon} ; \varepsilon\right)=t_{i}^{N} \quad \forall i=1, \ldots, N
$$

and the 'asymptotic expansion' $\bar{\mu}_{N}$ of $\mu_{N}$

$$
\bar{\mu}_{N}=\frac{1}{N} \sum_{i=1}^{N} \delta_{\bar{x}_{i}}
$$

with

$$
\bar{x}_{i}=x_{i}^{(0)}+\varepsilon x_{i}^{(1)}
$$

and

$$
\begin{aligned}
& x_{i}^{(0)}=A_{0}^{-1}\left(t_{i}^{N}\right) \\
& x_{i}^{(1)}=\frac{A_{1}\left(x_{i}^{(0)}\right)}{a_{0}\left(x_{i}^{(0)}\right)}
\end{aligned}
$$

Furthermore we denote by $\mu_{N}^{(0)}$ the discrete measure

$$
\mu^{(0)}=\frac{1}{N} \sum_{i=1}^{N} \delta_{x_{i}^{(0)}}
$$

With the notations given above we have the following theorems.

\section{Theorem 7}

$$
D\left(f, \mu_{N}\right)=D\left(\omega_{N}\right)
$$

Proof

Because the points $x_{i}^{\varepsilon}$ are the solutions of

$$
F(x ; \varepsilon)=t_{i}^{N}
$$

the theorem follows directly from the definition of the discrepancy.

\section{Corollary 1}

$$
D\left(f, \mu_{N}\right)=D\left(a_{0}, \mu_{N}^{(0)}\right)
$$

Concerning the discrepancy $D\left(f, \bar{\mu}_{N}\right)$ we get

Theorem 8 Assume $N \in \mathbb{N}$ fixed. Then there exists $h_{\varepsilon}$ with

$$
\frac{h_{\varepsilon}}{\varepsilon} \longrightarrow 0 \quad \text { if } \varepsilon \longrightarrow 0
$$

such that

$$
D\left(f, \bar{\mu}_{N}\right) \leq D\left(f, \mu_{N}\right)+h_{\varepsilon}
$$




\section{Proof}

From [5] we know that

$$
D\left(\omega_{N}\right)=\frac{1}{2 N}+\max _{i=1, \ldots, N}\left|\frac{2 i-1}{2 N}-t_{i}^{N}\right|
$$

if $\omega_{N}$ is a discrete measure on $[0,1]$ and $0 \leq t_{1}^{N} \leq t_{2}^{N} \leq \ldots \leq t_{N}^{N} \leq 1$.

Hence, using Theorem 7,

$$
D\left(f, \mu_{N}\right)=\frac{1}{2 N}+\max _{i=1, \ldots, N}\left|\frac{2 i-1}{2 N}-F\left(x_{i}^{\varepsilon} ; \varepsilon\right)\right|
$$

if $-\infty<x_{1}^{\varepsilon} \leq x_{2}^{\varepsilon} \leq \ldots \leq x_{N}^{\varepsilon}<\infty$ and

$$
D\left(f, \bar{\mu}_{N}\right) \leq D\left(f, \mu_{N}\right)+\max _{i=1, \ldots, N}\left|t_{i}^{N}-F\left(x_{i}^{(0)}+\varepsilon x_{i}^{(1)} ; \varepsilon\right)\right|
$$

Now

and with (101) we get

$$
t_{i}^{N}=A_{0}\left(x_{i}^{(0)}\right) \quad i=1, \ldots, N
$$

$$
D\left(f, \bar{\mu}_{N}\right) \leq D\left(f, \mu_{N}\right)+\max _{i=1, \ldots, N}\left|A_{0}\left(x_{i}^{(0)}\right)-F\left(x_{i}^{(0)}+\varepsilon x_{i}^{(1)} ; \varepsilon\right)\right|
$$

Because $F(x ; \varepsilon) \sim A_{0}(x)+\varepsilon A_{1}(x)$ (uniformly with respect to $x$ ) we have

$$
\begin{aligned}
D\left(f, \bar{\mu}_{N}\right) & \leq D\left(f, \mu_{N}\right) \\
& +\max _{i=1, \ldots, N}\left|A_{0}\left(x_{i}^{(0)}\right)-A_{0}\left(x_{i}^{(0)}+\varepsilon x_{i}^{(1)}\right)-\varepsilon A_{1}\left(x_{i}^{(0)}+\varepsilon x_{i}^{(1)}\right)\right| \\
& +k_{\varepsilon}
\end{aligned}
$$

and

$$
\frac{k_{\varepsilon}}{\varepsilon} \longrightarrow 0 \quad \text { if } \varepsilon \longrightarrow 0
$$

Both $A_{0}$ and $A_{1}$ are differentiable and

$$
\begin{aligned}
& A_{0}\left(x_{i}^{(0)}\right)-A_{0}\left(x_{i}^{(0)}+\varepsilon x_{i}^{(1)}\right)=\varepsilon x_{i}^{(1)} a_{o}\left(x_{i}^{(0)}\right)+h_{i}(\varepsilon) \\
& A_{1}\left(x_{i}^{(0)}\right)-A_{1}\left(x_{i}^{(0)}+\varepsilon x_{i}^{(1)}\right)=\varepsilon x_{i}^{(1)} a_{1}\left(x_{i}^{(0)}\right)+g_{i}(\varepsilon)
\end{aligned}
$$

with

$$
\begin{array}{ll}
\frac{h_{i}(\varepsilon)}{\varepsilon} \longrightarrow 0 & \text { if } \varepsilon \longrightarrow 0 \\
\frac{g_{i}(\varepsilon)}{\varepsilon} \longrightarrow 0 & \text { if } \varepsilon \longrightarrow 0
\end{array}
$$

for all $i=1, \ldots, N$.

Defining

$$
h_{\varepsilon}=\max _{i=1, \ldots, N}\left(h_{i}(\varepsilon)+\varepsilon^{2} x_{i}^{(1)} a_{1}\left(x_{i}^{(0)}\right)+\varepsilon g_{i}(\varepsilon)\right)+k_{\varepsilon}
$$

we have

$$
D\left(f, \bar{\mu}_{N}\right) \leq D\left(f, \mu_{N}\right)+h_{\varepsilon}
$$

and

which completes the proof.

$$
\frac{h_{\varepsilon}}{\varepsilon} \longrightarrow 0 \quad \text { if } \varepsilon \longrightarrow 0
$$


Corollary 2 Assume $N \in N$ fixed. Then there exists $\bar{h}_{\varepsilon}$ with

$$
\frac{\bar{h}_{\varepsilon}}{\varepsilon} \longrightarrow 0 \quad \text { if } \varepsilon \longrightarrow 0
$$

such that

$$
\left|\int_{R} \Phi f \mathrm{~d} x-\frac{1}{N} \sum_{i=1}^{N} \Phi\left(\bar{x}_{i}\right)\right| \leq V[\Phi] D\left(f, \mu_{N}\right)+\bar{h}_{\varepsilon}
$$

for all functions $\Phi: \mathbb{R} \longrightarrow \mathbb{R}$ which have bounded variation $V[\Phi]$ on $\mathbb{R}$.

Proof

The Corollary follows directly from Theorems 2 and 8 .

Furthermore we have the following result for the bounded Lipschitz distance between $f$ and $\bar{\mu}_{N}$ :

Theorem 9 Assume $N \in \mathbb{N}$ fixed. There exists $h_{\varepsilon}$ with

$$
\frac{h_{\varepsilon}}{\varepsilon} \longrightarrow 0 \quad \text { if } \varepsilon \longrightarrow 0
$$

such that

$$
\rho\left(f, \bar{\mu}_{N}\right) \leq \rho\left(f, \mu_{N}\right)+h_{\varepsilon}
$$

Proof

We first notice that, because $x_{i}^{\varepsilon} \sim x_{i}^{(0)}+\varepsilon x_{i}^{(1)}$, there exists functions $h_{i}(\varepsilon), i=1, \ldots, N$ with

$$
\frac{h_{i}(\varepsilon)}{\varepsilon} \longrightarrow 0 \quad \text { if } \varepsilon \longrightarrow 0
$$

such that

$$
x_{i}^{\varepsilon}=x_{i}^{(0)}+\varepsilon x_{i}^{(1)}+h_{i}(\varepsilon)
$$

Suppose $\Phi \in D=\{\Phi: \mathbb{R} \rightarrow \mathbb{R}, 0 \leq \Phi \leq 1,|\Phi(x)-\Phi(y)| \leq|x-y|\}$. Then

$$
\begin{aligned}
\left|\int_{R} \Phi f \mathrm{~d} x-\frac{1}{N} \sum_{i=1}^{N} \Phi\left(\bar{x}_{i}\right)\right| & \leq \rho\left(f, \mu_{N}\right)+\frac{1}{N} \sum_{i=1}^{N}\left|\Phi\left(x_{i}^{\varepsilon}\right)-\Phi\left(\bar{x}_{i}\right)\right| \\
& \leq \rho\left(f, \mu_{N}\right)+h_{\varepsilon}
\end{aligned}
$$

with

$$
h_{\varepsilon}=\max _{i=1, \ldots, N} h_{i}(\varepsilon)
$$

Corollary 3 Assume $N \in \mathbb{N}$ fixed. There exists $\bar{h}_{\varepsilon}$ with

$$
\frac{\bar{h}_{\varepsilon}}{\varepsilon} \longrightarrow 0 \quad \text { if } \varepsilon \longrightarrow 0
$$

such that

$$
\left|\int_{R} \Phi f \mathrm{~d} x-\frac{1}{N} \sum_{i=1}^{N} \Phi\left(\bar{x}_{i}\right)\right| \leq 2 \max \{L, M\} \rho\left(f, \mu_{N}\right)+\bar{h}_{\varepsilon}
$$

for all functions $\Phi: \mathbb{R} \longrightarrow \mathbb{R}$ which are bounded by $M<\infty$ and Lipschitz-continuous with Lipschitz constant $L$.

Proof

The Corollary follows directly from Theorems 4 and 9. 


\section{A Numerical Example}

We use the results of the previous section together with the following test problem:

$$
f(x ; \varepsilon)=\frac{1}{\sqrt{\pi}\left(1+\frac{15}{32} \varepsilon^{2}\right)}\left(1+\frac{\varepsilon}{2} x^{3}\right)^{2} \mathrm{e}^{-x^{2}}
$$

The density $f(x ; \varepsilon)$ given by $(123)$ is a simplification of the so-called modified Chapman-Enskog density [7]. This type of probability function is used in Computational Fluid Dynamics for the numerical coupling of the Navier-Stokes equations for continuum flows and the Boltzmann equation for rarefied gas flows.

One computes that

$$
\begin{gathered}
f(x ; \varepsilon) \sim \frac{1}{\sqrt{\pi}} \mathrm{e}^{-x^{2}}+\varepsilon \frac{x^{3}}{\sqrt{\pi}} \mathrm{e}^{-x^{2}} \\
F(x ; \varepsilon)=\frac{1}{2} \operatorname{erf}(x)+\frac{1}{2}-\frac{\varepsilon \mathrm{e}^{-x^{2}}}{\sqrt{\pi}\left(1+\frac{15}{32} \varepsilon^{2}\right)}\left(16+15 \varepsilon x+16 x^{2}+10 \varepsilon x^{3}+4 \varepsilon x^{5}\right)
\end{gathered}
$$

and

$$
F(x ; \varepsilon) \sim A_{0}(x)+\varepsilon A_{1}(x)
$$

with

$$
\begin{aligned}
& A_{0}(x)=\frac{1}{2}+\frac{1}{2} \operatorname{erf}(x) \\
& A_{1}(x)=-\frac{1}{2 \sqrt{\pi}}\left(1+x^{2}\right) \mathrm{e}^{-x^{2}}
\end{aligned}
$$

Using the result of section 3 we get for the asymptotic expansion of the solution $x^{\varepsilon}$ of $F(x ; \varepsilon)=t$

$$
\begin{aligned}
t & =\frac{1}{2}+\frac{1}{2} \operatorname{erf}\left(x^{(0)}\right) \\
x^{(1)} & =-\frac{1}{2}\left(\left(x^{(0)}\right)^{2}+1\right)
\end{aligned}
$$

and

$$
x^{\varepsilon} \sim x^{(0)}+\varepsilon x^{(1)}
$$

Table I shows the discrepancy errors using the optimal finite pointset $\omega_{N}^{o p t}$ on $[0,1]$ given by

$$
t_{i}^{N}=\frac{2 i-1}{2 N}
$$

with discrepancy $D\left(\omega_{N}^{o p t}\right)=\frac{1}{2 N}$.

Notation:

$$
\begin{aligned}
E\left(\mu_{N}\right) & =\left|D\left(\omega_{N}^{o p t}\right)-D\left(f, \mu_{N}\right)\right|=0 \\
E\left(\mu_{N}^{(0)}\right) & =\left|D\left(f, \mu_{N}\right)-D\left(f, \mu_{N}^{(0)}\right)\right| \\
E\left(\bar{\mu}_{N}\right) & =\left|D\left(f, \mu_{N}\right)-D\left(f, \bar{\mu}_{N}\right)\right|
\end{aligned}
$$


TAB. I. Discrepancy errors of $\bar{\mu}_{N}$ and $\mu_{N}^{(0)}$.

\begin{tabular}{|c|c|c|c|c|c|}
\hline$N$ & $D\left(\omega_{N}^{\text {opt }}\right)$ & $E\left(\mu_{N}^{(0)}\right)$ & $E\left(\mu_{N}^{(0)}\right)$ & $E\left(\mu_{N}^{(0)}\right)$ & $E\left(\mu_{N}^{(0)}\right)$ \\
\hline 10 & $5.0 \cdot 10^{-2}$ & $2.8704 \cdot 10^{-2}$ & $2.8230 \cdot 10^{-3}$ & $2.8211 \cdot 10^{-4}$ & $2.8209 \cdot 10^{-5}$ \\
100 & $5.0 \cdot 10^{-3}$ & $2.8770 \cdot 10^{-2}$ & $2.8241 \cdot 10^{-3}$ & $2.8211 \cdot 10^{-4}$ & $2.8210 \cdot 10^{-5}$ \\
1000 & $5.0 \cdot 10^{-4}$ & $2.8770 \cdot 10^{-2}$ & $2.8241 \cdot 10^{-3}$ & $2.8211 \cdot 10^{-4}$ & $2.8210 \cdot 10^{-5}$ \\
\hline & & $\varepsilon=1 \cdot 10^{-1}$ & $\varepsilon=1 \cdot 10^{-2}$ & $\varepsilon=1 \cdot 10^{-3}$ & $\varepsilon=1 \cdot 10^{-4}$ \\
\hline$N$ & $D\left(\omega_{N}^{\text {opt }}\right)$ & $E\left(\bar{\mu}_{N}\right)$ & $E\left(\bar{\mu}_{N}\right)$ & $E\left(\bar{\mu}_{N}\right)$ & $E\left(\bar{\mu}_{N}\right)$ \\
\hline 10 & $5.0 \cdot 10^{-2}$ & $2.1546 \cdot 10^{-3}$ & $2.0662 \cdot 10^{-5}$ & $2.0581 \cdot 10^{-7}$ & $2.0944 \cdot 10^{-9}$ \\
100 & $5.0 \cdot 10^{-3}$ & $2.1957 \cdot 10^{-3}$ & $2.0787 \cdot 10^{-5}$ & $2.0677 \cdot 10^{-7}$ & $2.0980 \cdot 10^{-9}$ \\
1000 & $5.0 \cdot 10^{-4}$ & $2.1964 \cdot 10^{-3}$ & $2.0790 \cdot 10^{-5}$ & $2.0677 \cdot 10^{-7}$ & $2.0991 \cdot 10^{-9}$ \\
\hline
\end{tabular}

One may recognize that

$$
\lim _{\varepsilon \rightarrow 0} E\left(\mu^{(0)}\right)=0
$$

and

$$
\lim _{\varepsilon \rightarrow 0} \frac{E\left(\bar{\mu}_{N}\right)}{\varepsilon}=0
$$

Now we take instead of $\omega_{N}^{o p t}$ a discrete measure $\omega_{N}$ generated via pseudo-random numbers on $[0,1]$. The random number generator is a linear-congruential method with parameters from the UNIX rand-subroutine.

TAB. II. Discrepancy of $\mu_{N}, \mu_{N}^{(0)}$ and $\bar{\mu}_{N}$ using pseudo-random numbers.

\begin{tabular}{|c|c|c|c|c|}
\hline$N$ & $D\left(f, \mu_{N}\right)$ & $D\left(f, \mu_{N}^{(0)}\right)$ & $D\left(f, \mu_{N}^{(0)}\right)$ & $D\left(f, \mu_{N}^{(0)}\right)$ \\
\hline 10 & $2.55126 \cdot 10^{-1}$ & $2.57716 \cdot 10^{-1}$ & $2.55123 \cdot 10^{-1}$ & $2.55124 \cdot 10^{-1}$ \\
100 & $8.48946 \cdot 10^{-2}$ & $9.34267 \cdot 10^{-2}$ & $8.49941 \cdot 10^{-2}$ & $8.48953 \cdot 10^{-2}$ \\
1000 & $2.67922 \cdot 10^{-2}$ & $4.56819 \cdot 10^{-2}$ & $2.70012 \cdot 10^{-2}$ & $2.67883 \cdot 10^{-2}$ \\
\hline & & $\varepsilon=1 \cdot 10^{-1}$ & $\varepsilon=1 \cdot 10^{-2}$ & $\varepsilon=1 \cdot 10^{-3}$ \\
\hline$N$ & $D\left(f, \mu_{N}\right)$ & $D\left(f, \bar{\mu}_{N}\right)$ & $D\left(f, \bar{\mu}_{N}\right)$ & $D\left(f, \bar{\mu}_{N}\right)$ \\
\hline 10 & $2.55126 \cdot 10^{-1}$ & $2.54742 \cdot 10^{-1}$ & $2.55122 \cdot 10^{-1}$ & $2.55126 \cdot 10^{-1}$ \\
100 & $8.48946 \cdot 10^{-2}$ & $8.48618 \cdot 10^{-2}$ & $8.48939 \cdot 10^{-2}$ & $8.48946 \cdot 10^{-2}$ \\
1000 & $2.67922 \cdot 10^{-2}$ & $2.68315 \cdot 10^{-2}$ & $2.67914 \cdot 10^{-2}$ & $2.67922 \cdot 10^{-2}$ \\
\hline
\end{tabular}

Remark 7 The convergence speed for the discrepancy using pseudo-random numbers is approximately $\frac{1}{\sqrt{N}}$.

Finally we investigate the integration error using the pointsets $\mu_{N}, \bar{\mu}_{N}$ and $\mu_{N}^{(0)}$ :

We take

$$
\mathbb{E}\left(x^{2}\right)=\int_{R} x^{2} f(x ; \varepsilon) \mathrm{d} x=\frac{32+105 \varepsilon^{2}}{64+30 \varepsilon^{2}}
$$


and denote by $E\left(\mu_{N}\right), E^{*}\left(\bar{\mu}_{N}\right)$ and $E^{*}\left(\mu_{N}^{(0)}\right)$ the following quantities

$$
\begin{aligned}
E\left(\mu_{N}\right) & =\left|\mathbb{E}\left(x^{2}\right)-\frac{1}{N} \sum_{i=1}^{N}\left(x_{i}^{\varepsilon}\right)^{2}\right| \\
E^{*}\left(\mu_{N}\right) & =\left|\frac{1}{N} \sum_{i=1}^{N}\left[\left(x_{i}^{\varepsilon}\right)^{2}-\bar{x}_{i}^{2}\right]\right| \\
E^{*}\left(\mu_{N}^{(0)}\right) & =\left|\frac{1}{N} \sum_{i=1}^{N}\left[\left(x_{i}^{\varepsilon}\right)^{2}-\left(x_{i}^{(0)}\right)^{2}\right]\right|
\end{aligned}
$$

The function $\Phi(x)=x^{2}$ is not Lipschitz-continuous and has unbounded variation on $\mathbb{R}$ - hence one can not apply Theorem 9 or Corollary 4 to estimate the integration error.

We again take first the pointset $\omega_{N}^{o p t}$ to compute $\mu_{N}$ respectively $\mu_{N}^{(0)}$. The results are shown in table 3 .

TAB. III. Integration error for $\mathbb{E}\left(x^{2}\right)$ using the optimal pointset $\omega_{N}^{o p t}$.

\begin{tabular}{|c|c|c|c|c|}
\hline$N$ & $\varepsilon$ & $E\left(\mu_{N}\right)$ & $E^{*}\left(\bar{\mu}_{N}\right)$ & $E^{*}\left(\mu_{N}^{(0)}\right)$ \\
\hline 10 & 0.1 & $6.0721 \cdot 10^{-2}$ & $7.5954 \cdot 10^{-3}$ & $1.3383 \cdot 10^{-2}$ \\
10 & 0.01 & $6.0112 \cdot 10^{-2}$ & $7.7206 \cdot 10^{-5}$ & $1.3508 \cdot 10^{-4}$ \\
\hline 100 & 0.1 & $6.0979 \cdot 10^{-3}$ & $7.5492 \cdot 10^{-3}$ & $1.4244 \cdot 10^{-2}$ \\
100 & 0.01 & $6.3409 \cdot 10^{-3}$ & $7.7921 \cdot 10^{-5}$ & $1.4487 \cdot 10^{-4}$ \\
100 & 0.001 & $6.3451 \cdot 10^{-3}$ & $7.7947 \cdot 10^{-7}$ & $1.4490 \cdot 10^{-6}$ \\
\hline 1000 & 0.1 & $6.0226 \cdot 10^{-4}$ & $7.1951 \cdot 10^{-3}$ & $1.4045 \cdot 10^{-2}$ \\
1000 & 0.01 & $6.4869 \cdot 10^{-4}$ & $7.3504 \cdot 10^{-5}$ & $1.4200 \cdot 10^{-4}$ \\
1000 & 0.001 & $6.5006 \cdot 10^{-4}$ & $7.3519 \cdot 10^{-7}$ & $1.4202 \cdot 10^{-6}$ \\
\hline 10000 & 0.1 & $7.7054 \cdot 10^{-5}$ & $7.1137 \cdot 10^{-3}$ & $1.3985 \cdot 10^{-2}$ \\
10000 & 0.01 & $6.5300 \cdot 10^{-5}$ & $7.2185 \cdot 10^{-5}$ & $1.4090 \cdot 10^{-4}$ \\
10000 & 0.001 & $6.5581 \cdot 10^{-5}$ & $7.2193 \cdot 10^{-7}$ & $1.4091 \cdot 10^{-6}$ \\
\hline
\end{tabular}

With the same notation we have the following results using pseudo-random numbers. 
TAB. IV. Integration error for $\mathbb{E}\left(x^{2}\right)$ using pseudo-random numbers.

\begin{tabular}{|c|c|c|c|c|}
\hline$N$ & $\varepsilon$ & $E\left(\mu_{N}\right)$ & $E^{*}\left(\bar{\mu}_{N}\right)$ & $E^{*}\left(\mu_{N}^{(0)}\right)$ \\
\hline 10 & 0.1 & $1.8532 \cdot 10^{-1}$ & $7.7183 \cdot 10^{-3}$ & $4.4908 \cdot 10^{-2}$ \\
\hline 100 & 0.1 & $5.7927 \cdot 10^{-2}$ & $7.1100 \cdot 10^{-3}$ & $1.9255 \cdot 10^{-2}$ \\
100 & 0.01 & $5.4788 \cdot 10^{-2}$ & $7.2687 \cdot 10^{-5}$ & $1.5441 \cdot 10^{-3}$ \\
\hline 1000 & 0.1 & $1.8476 \cdot 10^{-2}$ & $7.1336 \cdot 10^{-3}$ & $1.4041 \cdot 10^{-2}$ \\
1000 & 0.01 & $1.7312 \cdot 10^{-2}$ & $7.1719 \cdot 10^{-5}$ & $5.0428 \cdot 10^{-4}$ \\
\hline 10000 & 0.1 & $5.5549 \cdot 10^{-3}$ & $7.1181 \cdot 10^{-3}$ & $1.4049 \cdot 10^{-2}$ \\
10000 & 0.01 & $5.3119 \cdot 10^{-3}$ & $7.1778 \cdot 10^{-5}$ & $2.0086 \cdot 10^{-4}$ \\
10000 & 0.001 & $5.3150 \cdot 10^{-3}$ & $7.1786 \cdot 10^{-7}$ & $1.5753 \cdot 10^{-5}$ \\
\hline
\end{tabular}

\section{References}

[1] N. Bleistein and R.A. Handelsmann, Asymptotic expansions of integrals, Holt, Rinehart and Winston, New York (1975).

[2] L. Devroye, Non-Uniform Random Variate Generation, Springer Verlag, New York (1986).

[3] R. Dudley, Probabilities and Metrics, Aarhus Universiteit, Lecture Notes Series No. 45 (1976).

[4] H. Neunzert and J. Wick, Die Darstellung von Funktionen mehrerer Variablen durch Punktmengen,(in German), Report No. 996, KFA Jülich (1975).

[5] H. Niederreiter, Random Number Generation and Quasi-Monte Carlo Methods, SIAM, Philadelphia (1993).

[6] F.W.J. Olver, Aymptotics and special functions, Academic Press, New York (1974).

[7] W.G. Vincenti and C.H. Kruger, Introduction to Physical Gas Dynamics, Krieger Publishing Company, Huntington (1975).

Jens Struckmeier

Department of Mathematics

University of Kaiserslautern

P.O. Box 3049

67653 Kaiserslautern

Germany

E-Mail: struckm@mathematik.uni-kl.de 